\title{
Mechanisms of exercise limitation in patients with chronic hypersensitivity pneumonitis
}

\author{
Olívia Meira Dias (1)', Bruno Guedes Baldi ${ }^{1}$, Jeferson George Ferreira', \\ Letícia Zumpano Cardenas ${ }^{1}$, Francesca Pennati ${ }^{2}$, Caterina Salito ${ }^{2}$, \\ Carlos Roberto Ribeiro Carvalho ${ }^{1}$, Andrea Aliverti ${ }^{2}$ and \\ André Luis Pereira de Albuquerque ${ }^{1}$
}

Affiliations: ${ }^{1}$ Divisao de Pneumologia, Instituto do Coracao (InCor), Hospital das Clinicas HCFMUSP, Faculdade de Medicina, Universidade de Sâo Paulo, Sâo Paulo, Brazil. ${ }^{2}$ Dipartimento di Elettronica, Informazione e Bioingegneria, Politecnico di Milano, Milan, Italy.

Correspondence: Olívia Meira Dias, Divisao de Pneumologia, Instituto do Coracao (InCor), Hospital das Clinicas HCFMUSP, Faculdade de Medicina, Universidade de Sao Paulo, Dr Enéas de Carvalho Aguiar 44, São Paulo, 05409002, Brazil. E-mail: meiradiasđyahoo.com.br

ABSTRACT Small airway and interstitial pulmonary involvements are prominent in chronic hypersensitivity pneumonitis (cHP). However, their roles on exercise limitation and the relationship with functional lung tests have not been studied in detail.

Our aim was to evaluate exercise performance and its determinants in cHP. We evaluated maximal cardiopulmonary exercise testing performance in $28 \mathrm{cHP}$ patients (forced vital capacity $57 \pm 17 \%$ pred) and 18 healthy controls during cycling.

Patients had reduced exercise performance with lower peak oxygen production (16.6 (12.3-19.98) $\mathrm{mL} \cdot \mathrm{kg}^{-1} \cdot \mathrm{min}^{-1}$ versus 25.1 (16.9-32.0), $\left.\mathrm{p}=0.003\right)$, diminished breathing reserve (\% maximal voluntary ventilation) (12 (6.4-34.8)\% versus $41(32.7-50.8) \%, \mathrm{p}<0.001)$ and hyperventilation (minute ventilation/ carbon dioxide production slope $37 \pm 5$ versus $31 \pm 4, \mathrm{p}<0.001$ ). All patients presented oxygen desaturation and augmented Borg dyspnoea scores (8 (5-10) versus $4(1-7), \mathrm{p}=0.004)$. The prevalence of dynamic hyperinflation was found in only $18 \%$ of patients. When comparing cHP patients with normal and low peak oxygen production ( $<84 \%$ pred, lower limit of normal), the latter exhibited a higher minute ventilation/carbon dioxide production slope $(39 \pm 5.0$ versus $34 \pm 3.6, p=0.004)$, lower tidal volume $(0.84$ (0.78-0.90) L versus $1.15(0.97-1.67) \mathrm{L}, \mathrm{p}=0.002)$, and poorer physical functioning score on the Short form-36 health survey. Receiver operating characteristic curve analysis showed that reduced lung volumes (forced vital capacity \%, total lung capacity \% and diffusing capacity of the lung for carbon dioxide \%) were high predictors of poor exercise capacity.

Reduced exercise capacity was prevalent in patients because of ventilatory limitation and not due to dynamic hyperinflation. Reduced lung volumes were reliable predictors of lower performance during exercise.

@ERSpublications

Besides significant small airway involvement, reduced exercise capacity is due to ventilatory limitation and not due to dynamic hyperinflation in chronic hypersensitivity pneumonitis http://ow.ly/Ou9230kSBQz

Cite this article as: Dias OM, Baldi BG, Ferreira JG, et al. Mechanisms of exercise limitation in patients with chronic hypersensitivity pneumonitis. ERJ Open Res 2018; 4: 00043-2018 [https:// doi.org/10.1183/23120541.00043-2018].

This article has supplementary material available from openres.ersjournals.com

This study is registered at www.ClinicalTrials.gov with identifier number NCT 02523833.

Received: March 122018 | Accepted after revision: July 052018

Copyright $\odot$ ERS 2018. This article is open access and distributed under the terms of the Creative Commons Attribution Non-Commercial Licence 4.0. 


\section{Introduction}

Hypersensitivity pneumonitis is an interstitial lung disease (ILD) with variable clinical symptoms, caused by inhalation of specific organic antigens or low-molecular weight substances in genetically susceptible individuals [1-4]. Chronic hypersensitivity pneumonitis (cHP) represents the final stage of the disease, in which prolonged antigenic exposure causes lung fibrosis.

A unique feature of hypersensitivity pneumonitis is some degree of abnormality within and/or around the small airways, observed to be present in all patients with hypersensitivity pneumonitis in histopathology [5] and in some patients when evaluated by pulmonary functional tests (PFTs) [6]. Computed tomography (CT) scans have provided evidence for small airway disease in $\mathrm{cHP}$, suggested by mosaic attenuation pattern, air trapping on expiratory scans, centrilobular ground-glass opacities and the absence of predominance in inferior lobes [7-9].

Few studies have assessed exercise capacity in cHP patients [10, 11]; however, the mechanisms underlying these exercise limitations were not properly evaluated. One study evaluating patients with farmer's lung acutely exposed to mould showed an increased alveolar-arterial gradient, increased dead space and decreased ventilatory reserve at the end of cardiopulmonary exercise testing (CPET) [11]. Another investigation evaluated the prevalence of pulmonary hypertension in $\mathrm{CHP}$ patients using haemodynamic evaluation, and CPET was performed as a complimentary test [10]. Oxygen uptake $\left(V^{\prime} \mathrm{O}_{2}\right)$ at peak exercise and the anaerobic threshold were significantly lower in patients with pulmonary hypertension; however, no significant differences were found in any other CPET parameter [10]. Since cHP is initially characterised by airway involvement and later by interstitial fibrosis, exercise limitations may potentially be secondary to: 1) airway obstruction with dynamic hyperinflation (DH); and/or 2) significant oxygen desaturation and small tidal volume $(V \mathrm{~T})$ increment.

The presence of $\mathrm{DH}$ has been confirmed in several studies in moderate-to-severe chronic obstructive pulmonary disease (COPD) and is strongly related to augmented dyspnoea [12-14]. DH is also prevalent in lymphangioleiomyomatosis (LAM), an ILD, and is associated with exercise limitations and increased breathlessness [15]. In other ILDs, small numbers of studies have indicated that inspiratory capacity (IC) throughout exercise remains largely unaltered $[16,17]$, while others have found expiratory flow limitation during exercise [18]. Elucidating the prevalent mechanisms underlying exercise limitations in cHP might have a large impact on treatment decisions.

Therefore, we aimed to: 1) determine the exercise capacity and mechanisms of exercise impairment in cHP patients compared to healthy subjects; 2) evaluate the presence of DH through IC manoeuvers during maximal incremental CPET; and 3) compare cHP patients with normal and impaired exercise performance and investigate predictive functional values for worse exercise response.

\section{Materials and methods}

\section{Study subjects}

This study is part of a comprehensive study evaluating small airway involvement in cHP patients (www. ClinicalTrials.gov identifier NCT 02523833) with volumetric CT scans, PFTs and CPET. From September 2015 to March 2017, 178 patients with a confirmed cHP diagnosis, followed at the ILD outpatient clinic of the Pulmonary Division of the Hospital das Clínicas of the University of São Paulo, São Paulo, Brazil, were prospectively evaluated and 28 were enrolled (figure 1). cHP diagnosis was defined on CT findings (fibrotic involvement of the pulmonary parenchyma), known antigenic exposure, exclusion of other possible diagnoses, and compatible histology upon transbronchial or open lung biopsy, or bronchoalveolar lavage with lymphocytosis $>30 \%$. Exclusion criteria are detailed in the supplemental material. 18 age-matched healthy, nonsmoking subjects (mean age: $54 \pm 12.8$ years, forced vital capacity (FVC) $89 \%$

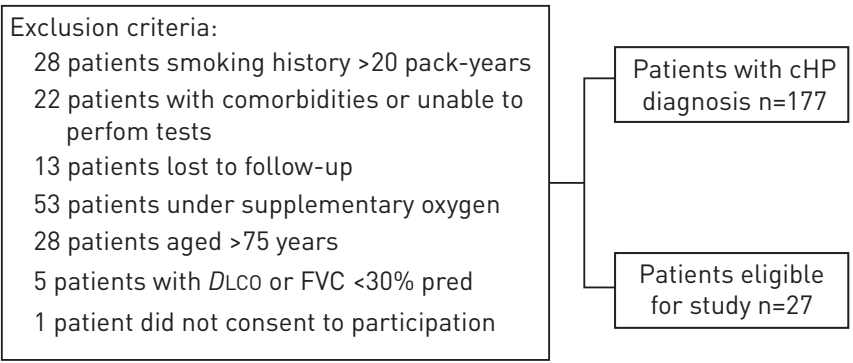

FIGURE 1 Study recruitment protocol. cHP: chronic hypersensitivity pneumonitis; DLco: diffusing capacity of the lung for carbon monoxide; FVC: forced vital capacity. 
predicted) were also studied as a control group. The study protocol was approved by the local research ethics committee (SDC 3966/13/091), and all patients signed informed consent.

\section{Measurements}

Dyspnoea and quality of life were assessed using the modified Baseline Dyspnoea Index and the Short form-36 health survey (SF-36) questionnaire, which has been validated for the Brazilian population [19, 20].

\section{Pulmonary function tests}

PFTs were performed following European Respiratory Society/American Thoracic Society guidelines [21-23]. Spirometry was performed using a calibrated pneumotachograph (Medical Graphics Corporation, St. Paul, MN, USA). Lung volumes and diffusing capacity of the lung for carbon dioxide (DLCO) were measured with a body plethysmograph (Elite Dx, Elite Series; Medical Graphics Corporation). FVC, forced expiratory volume in $1 \mathrm{~s}$ (FEV1), total lung capacity (TLC), residual volume (RV), and DLCO were obtained. Subsequently, all patients received salbutamol $(400 \mu \mathrm{g})$ and repeated the same variables measured in baseline spirometry. Predicted values were derived from the Brazilian population [24, 25].

\section{Cardiopulmonary exercise testing}

All patients underwent incremental, ramp type CPET on a cycle ergometer (model VIA Sprint 150P; CareFusion, San Diego, CA, USA), up to the tolerance limit or if presented any reason for interruption. The CPET protocol consisted of a symptom-limited incremental exercise test, including 2 min without pedalling, a warm-up period of 2 min (free wheel (FW)) followed by a progressively increasing work rate in a ramp fashion $\left(5-15 \mathrm{~W} \cdot \mathrm{min}^{-1}\right)$, and then a 3 -min recovery phase. The incremental rate was selected according to the physical fitness and pulmonary functional evaluation of each patient, aiming at test duration of 8-12 min.

The system used was the Vmax Encore CPET (VIASYS CareFusion), which is composed of a gas analyser coupled to a flow module through a mouthpiece. An integrated 12-lead electrocardiogram (Cardio Perfect PC-based 12 lead ECG; Welch Allyn, INC., Skaneateles Falls, NY, USA) was registered and peripheral oxygen saturation, using the NONIN oximeter (Onyx model 9500; Nonin Medical, INC., Plymouth, MN, USA), was continuously measured. The reference values used are those proposed for the Brazilian population by NEDER et al. [26]. Aerobic capacity was considered normal if values of $V^{\prime} \mathrm{O}_{2}$ max were $\geqslant 84 \%$ pred [27]. Discomfort in the lower limbs and dyspnoea intensity were assessed before the test, every $2 \mathrm{~min}$ and at the end of the test using the modified Borg scale [28]. Maximal exercise was determined if patients reached $85 \%$ of predicted maximal heart rate, had no ventilatory reserve or significant oxygen desaturation $(<80 \%)$ or respiratory exchange rate $>1.15$.

Measured variables and test interruption criteria are detailed in the supplemental material. To evaluate the presence of $\mathrm{DH}$, the IC was measured at the end of expiration at rest and every $2 \mathrm{~min}$. The end expiratory lung volume was obtained indirectly by the difference in the TLC by the IC $[29,30]$. Two reproducible manoeuvers were performed at each moment, with a difference $<150 \mathrm{~mL}$, and the highest value was adopted. The occurrence of $\mathrm{DH}$ was defined as IC variations of at least $10 \%$ from baseline [30].

\section{Statistical analysis}

Data are presented as mean \pm SD for normally distributed variables or as median (25th-75th percentiles) for non-normally distributed variables. Unpaired t-tests or the Mann-Whitney U-test were used to compare continuous variables between normal subjects and cHP patients, and in-between cHP patients.

A two-way repeated measures ANOVA was used to analyse time course differences of the variables with normal distribution and to evaluate differences between groups. The receiver operating characteristic (ROC) curve was used to identify lung function parameters (\% predicted) with the best accuracy for predicting reduced exercise performance $\left(V^{\prime} \mathrm{O}_{2}<84 \%\right.$ pred) in cHP patients. Significance was set at $\mathrm{p}<0.05$. Statistical analyses were performed using SPSS version 21.0 (IBM Corp, Armonk, NY, USA).

\section{Results}

\section{Baseline patient characteristics}

The general characteristics of cHP patients and healthy subjects paired by age, sex, and body mass index are presented in table 1 . All but four cHP patients had restrictive lung disease; however, all had reduced DLCO. Only three patients had a RV/TLC ratio $>0.40$ plus RV $>140 \%$ pred, consistent with concomitant air trapping. None had bronchodilator response to salbutamol. At the time of the study, 18 patients were receiving treatment and 10 had previously received immunosuppressors. Additionally, 25 (89\%) received or were receiving corticosteroids, and $13(46 \%)$ received or were receiving cytotoxic drugs.

Pulmonary biopsy was performed in $78 \%$ of patients and bronchoalveolar lavage in $75 \%$. From biopsied patients, 17 underwent transbronchial biopsy. The main findings were chronic lymphocytic bronchiolitis 


\begin{tabular}{|c|c|c|}
\hline & cHP & Healthy subjects \\
\hline Subjects $n$ & 28 & 18 \\
\hline Females & $16(57.1)$ & $10(55.6)$ \\
\hline Age years & $56 \pm 11.5$ & $54 \pm 12.8$ \\
\hline BMI $\mathrm{kg} \cdot \mathrm{m}^{-2}$ & $27.2(25.4-30.4)$ & $26.6(23-28)$ \\
\hline Baseline dyspnoea index mMRC & $1(1-2)$ & \\
\hline Smokers & $8(29)$ & \\
\hline Oxygen saturation at rest $\%$ & $94 \pm 2$ & \\
\hline \multicolumn{3}{|l|}{ Symptoms at diagnosis } \\
\hline Dyspnoea & $24(86)$ & \\
\hline Cough & $23(82)$ & \\
\hline Wheezing & $20(71)$ & \\
\hline Weight loss & $11(38)$ & \\
\hline Duration of symptoms months & $24(12-45)$ & \\
\hline \multicolumn{3}{|l|}{ Exposure } \\
\hline Mould & $21(75)$ & \\
\hline Birds & $18(64)$ & \\
\hline Feather pillow & $6(21)$ & \\
\hline Others & $3(11)$ & \\
\hline No recognised exposure & $0(0)$ & \\
\hline Actual exposure/previous exposure $n$ & $4 / 24$ & \\
\hline \multicolumn{3}{|l|}{ Pulmonary function tests } \\
\hline FVC L & $2.03 \pm 0.65$ & $3.33 \pm 0.85^{*}$ \\
\hline FVC \% pred & $57 \pm 17$ & $89 \pm 10^{*}$ \\
\hline FEV $1 \mathrm{~L}$ & $1.75 \pm 0.52$ & $2.74 \pm 0.62 *$ \\
\hline FEV $1 \%$ pred & $61 \pm 16$ & $90 \pm 10 *$ \\
\hline $\mathrm{FEV}_{1} / \mathrm{FVC}$ & $0.87 \pm 0.05$ & $0.82 \pm 0.04^{*}$ \\
\hline FEF $25-75 \%$ & $98 \pm 31$ & \\
\hline TLC L & $3.37 \pm 0.90$ & \\
\hline TLC \% pred & $64(53-72)$ & \\
\hline RV L & $1.18(1.06-1.47)$ & \\
\hline RV \% pred & $77(60-88)$ & \\
\hline $\mathrm{RV} / \mathrm{TLC}$ & $0.39 \pm 0.07$ & \\
\hline DLco \% pred & $42(31-63)$ & \\
\hline \multicolumn{3}{|l|}{ HRCT findings } \\
\hline Centrilobular nodules & $3(11)$ & \\
\hline Ground-glass opacities & $26(96)$ & \\
\hline Mosaic pattern & $15(54)$ & \\
\hline Honeycombing & $4(14)$ & \\
\hline Findings suggestive of fibrosis & 25 (89) & \\
\hline Emphysema & $0(0)$ & \\
\hline Bronchoalveolar lavage & $21(75)$ & \\
\hline Lymphocyte count & $23(10-38)$ & \\
\hline CD4/CD8 ratio & $0.57(0.37-1.29)$ & \\
\hline Lung biopsy & $21(78)$ & \\
\hline Transbronchial & $16(59)$ & \\
\hline Surgical & $5(19)$ & \\
\hline
\end{tabular}

Data are presented as $\mathrm{n}(\%)$, mean \pm SD if normally distributed or median (25th-75th percentile), unless otherwise stated. BMI: body mass index; mMRC: modified Medical Research Council; FVC: forced vital capacity; FEV1: forced expiratory volume in $1 \mathrm{~s}$; FEF25-75\%: forced expiratory flow at $25-75 \%$ of FVC; TLC: total lung capacity; RV: residual volume; DLCO: diffusing capacity of the lung for carbon monoxide; HRCT: high-resolution computed tomography. *: $p<0.05$.

$(n=13)$, bronchiolar metaplasia $(n=2)$, classic pattern with chronic cellular bronchiolitis and the presence of malformed granulomas $(n=1)$; in one transbronchial biopsy, material was not representative. Among five patients that underwent open lung biopsy, four patients had nonspecific interstitial pneumonia pattern associated with bronchiolocentric fibrotic involvement and one patient presented the classic histological hypersensitivity pneumonitis triad (chronic interstitial pneumonia with peribronchiolar accentuation, bronchiolitis and non-caseating granulomas). 


\section{CPET for healthy subjects and cHP patients}

Table 2 shows CPET data at peak exercise. cHP patients had diminished exercise performance (workload and oxygen consumption) compared to healthy subjects. Considering the ventilatory responses, the final breathing reserve (\% maximal voluntary ventilation) was significantly reduced in $\mathrm{cHP}$ patients and they also demonstrated a greater respiratory rate with lower $V \mathrm{~T}$, in the presence of increased ventilatory response (higher minute ventilation $\left(V^{\prime} \mathrm{E}\right) /$ carbon dioxide production $\left(V^{\prime} \mathrm{CO}_{2}\right)$ slope) during exercise. Concerning cardiovascular responses, cHP patients achieved lower heart rates and obtained lower oxygen pulse. However, no patient presented evidence of cardiovascular limitations, including pulmonary hypertension with early plateau in oxygen pulse or a significant decrease in end-tidal carbon dioxide tension ( $\mathrm{mmHg}$ ) during the test. Finally, all but two cHP patients presented significant oxygen desaturation during exercise (desaturation $>4 \%$ compared to baseline levels), and $46 \%$ interrupted the CPET due to significant desaturation (arterial oxygen saturation $<80 \%$ ). Increased breathlessness (augmented Borg dyspnoea score) and dyspnoea (93\%) were the major reasons for CPET interruption.

Figure 2 illustrates $V^{\prime} \mathrm{E}, V_{\mathrm{T}}$ and dyspnoea (Borg scale) responses paired to workload during the exercise test in cHP patients compared with controls. $V^{\prime} \mathrm{E}$ was significantly higher in controls when compared with patients for all workloads $(\mathrm{p}<0.05)$. VT also significantly differed during $\mathrm{FW}, 40 \mathrm{~W}$ and at peak exercise $(p<0.05)$. Dyspnoea scores were higher in cHP patients; however, this difference was not statistically significant. No patient presented complications resulting in early interruption of the test.

Only one cHP patient was unable to perform acceptable IC manoeuvers according to the protocol and thus was excluded from the analysis. No statistically significant difference in IC variation between healthy and cHP patients was observed. Regarding the remaining 27 patients during CPET, five (18\%) had an IC decrease $>10 \%$ during exercise, which is consistent with $\mathrm{DH}$, whereas this was not observed in any control participant. The IC variation in relation to workload at peak effort compared to baseline levels in percentage and litres is illustrated in figure E1. We found that three cHP patients with $\mathrm{DH}$ had normal exercise capacity and only two had lower exercise capacity.

TABLE 2 Incremental cardiopulmonary exercise testing on a cycle ergometer (peak exercise data) in patients with chronic hypersensitivity pneumonitis (cHP) and healthy subjects

\begin{tabular}{|c|c|c|c|}
\hline & $\mathrm{cHP}$ & Healthy subjects & p-value \\
\hline Subjects $\mathbf{n}$ & 28 & 18 & \\
\hline Exercise time $\min$ & $7.24 \pm 3.02$ & $7.17 \pm 2.05$ & 0.89 \\
\hline RER & $1.04 \pm 0.14$ & $1.08 \pm 0.13$ & 0.30 \\
\hline Work rate $\mathbf{W}$ & $66 \pm 35$ & $138 \pm 69$ & $<0.001$ \\
\hline Work rate $\%$ pred & $53 \pm 23$ & $104 \pm 35$ & $<0.001$ \\
\hline$V^{\prime} \mathrm{O}_{2} \mathrm{~mL} \cdot \mathbf{k g}^{-1} \cdot \mathrm{min}^{-1}$ & $16.6(12.3-19.98)$ & $25.1(16.9-32.0)$ & 0.003 \\
\hline$V^{\prime} \mathrm{O}_{2} \%$ pred & $83(63-97)$ & $112(89-118)$ & $<0.001$ \\
\hline$V^{\prime} \mathrm{E} L \cdot \mathrm{min}^{-1}$ & $50(42-63)$ & $71(45-89)$ & 0.05 \\
\hline Breathing reserve $\%$ MVV & $12(6.4-34.8)$ & $41(32.7-50.8)$ & $<0.001$ \\
\hline$V^{\prime} \mathrm{E} / V^{\prime} \mathrm{CO}_{2}$ slope & $37 \pm 5$ & $31 \pm 4$ & $<0.001$ \\
\hline V $\mathrm{L}$ & $0.91(0.82-1.25)$ & $1.75(1.29-2.21)$ & $<0.001$ \\
\hline Respiratory rate breaths $\cdot \min ^{-1}$ & $52 \pm 11$ & $40 \pm 10$ & 0.001 \\
\hline$V$ V/IC & $0.77 \pm 0.15$ & $0.68 \pm 0.16$ & 0.06 \\
\hline$\Delta P_{\mathrm{ETCO}} \mathrm{mmHg}$ & $-0.24 \pm 2.67$ & $-0.11 \pm 2.83$ & 0.88 \\
\hline HR beats $\cdot \mathrm{min}^{-1}$ & $133 \pm 20$ & $149 \pm 26$ & 0.03 \\
\hline HR \% pred & $84(75-89)$ & 95 (88-99) & 0.008 \\
\hline $\mathrm{V}^{\prime} \mathrm{O}_{2} / \mathrm{HR} \mathrm{mL} \cdot$ beat $^{-1} \cdot \mathrm{min}^{-1}$ & $9.5 \pm 2.9$ & $12.6 \pm 5.0$ & 0.01 \\
\hline$V^{\prime} \mathrm{O}_{2} / \mathrm{HR} \%$ pred & $93 \pm 19$ & $122 \pm 29$ & $<0.001$ \\
\hline $\mathrm{SpO}_{2} \%$ & $81 \pm 7$ & $95 \pm 2$ & $<0.001$ \\
\hline Borg dyspnoea score & $8(5-10)$ & $4(1-7)$ & 0.004 \\
\hline Borg leg discomfort score & $8(4-9)$ & $7(4-9)$ & 0.71 \\
\hline$\Delta I C$ from rest $L$ & $-0.01 \pm 0.20$ & $0.11 \pm 0.25$ & 0.08 \\
\hline$\Delta \mathrm{IC}$ from rest $\%$ & $1.92 \pm 12$ & $4.96 \pm 11$ & 0.43 \\
\hline
\end{tabular}

Data are presented as mean \pm SD or median (25th-75th percentile), unless otherwise stated. RER: respiratory exchange rate; $V^{\prime} \mathrm{O}_{2}$ : oxygen production; $V^{\prime} \mathrm{E}$ : minute ventilation; $\mathrm{MVV}$ : maximal voluntary ventilation; $V^{\prime} \mathrm{CO}_{2}$ : carbon dioxide production; $V \mathrm{~T}$ : tidal volume; IC: inspiratory capacity; $P \mathrm{ETCO}_{2}$ : end-tidal carbon dioxide tension; $\mathrm{HR}$ : heart rate; $\mathrm{SpO}_{2}$ : arterial oxygen saturation measured by pulse oximetry. 

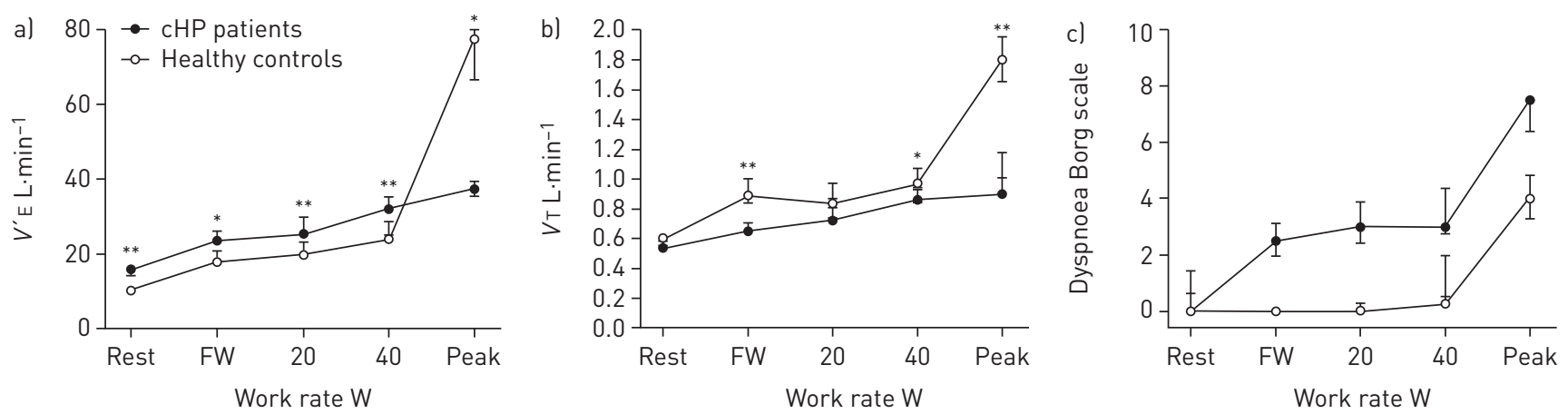

FIGURE 2 a) Minute ventilation ( $\left.V^{\prime} E\right)$, b) tidal volume $(V T)$ and $c$ ) dyspnoea (Borg score) paired to workload during an incremental cycle exercise test in patients with chronic hypersensitivity pneumonitis (CHP) compared to age-matched healthy controls. $V^{\prime} E$ and dyspnoea intensity were higher in cHP patients at any given work rate, whereas VT was higher in controls at free wheel (FW), $40 \mathrm{~W}$ and peak exercise. Dyspnoea scores were higher in cHP patients, although this difference was not significant. Data are presented as mean \pm SEM. ${ }^{*}$ : $p<0.05, * *$ : $p<0.005$ for cHP patients versus controls.

Exercise data of cHP patients with normal and low exercise capacity

Table 3 shows CPET data at peak exercise comparing cHP patients to controls $\left(V^{\prime} \mathrm{O}_{2} \geqslant 84 \%\right.$ pred) and low peak $V^{\prime} \mathrm{O}_{2}$. Dyspnoea interrupted exercise in all but two patients in the normal exercise capacity subgroup. As expected, patients with low peak $V^{\prime} \mathrm{O}_{2}$ had lower exercise performance, and also significantly higher $V^{\prime} \mathrm{E} / V^{\prime} \mathrm{CO}_{2}$ slope and lower $V \mathrm{~T}$. Differences were also observed in this subgroup with reduced oxygen pulse and heart rate. No differences were seen in all other exercise variables, including IC variation and VT/IC.

Patients with normal exercise capacity had more preserved lung volumes, FEV1 and DLCO (table 4). The ROC curve analysis showed that the FVC (\% pred) with the highest accuracy to predict reduced exercise capacity was 58.5\% (area under the curve (AUC) 0.824, 95\% CI 0.67-0.98, p=0.004; sensitivity and specificity of 78.6\%). Also, a TLC (\% pred) of 58.5\% (AUC 0.806, 95\% CI 0.64-0.97,

\section{TABLE 3 Incremental cardiopulmonary exercise on a cycle ergometer (peak exercise data) in patients with chronic hypersensitivity pneumonitis}

\begin{tabular}{|c|c|c|c|}
\hline & $V^{\prime} 0_{2} \geqslant 84 \%$ pred & $V^{\prime} \mathrm{O}_{2}<84 \%$ pred & p-value \\
\hline Subjects $n$ & 14 & 14 & \\
\hline Exercise time $\min$ & $8.4 \pm 2.4$ & $6.0 \pm 2.5$ & 0.02 \\
\hline Work rate $\mathrm{W}$ & $83.4 \pm 36$ & $47.86 \pm 24$ & 0.005 \\
\hline Work rate \%pred & $70.0 \pm 17$ & $36.0 \pm 15$ & $<0.001$ \\
\hline$V^{\prime} \mathrm{O}_{2} \mathrm{~mL} \cdot \mathrm{min}^{-1} \cdot \mathrm{kg}^{-1}$ & $20.51 \pm 6.42$ & $20.51 \pm 6.42$ & 0.006 \\
\hline$V^{\prime} \mathrm{O}_{2} \%$ pred & $99 \pm 12$ & $63 \pm 11$ & $<0.001$ \\
\hline$\Delta P \mathrm{ETCO}_{2} \mathrm{mmHg}$ & $0.06 \pm 2.39$ & $-0.53 \pm 2.99$ & 0.56 \\
\hline RER & $1.04 \pm 0.12$ & $1.03 \pm 0.16$ & 0.81 \\
\hline$V^{\prime} \mathrm{E} / V^{\prime} \mathrm{CO}_{2}$ slope & $34 \pm 3.6$ & $39 \pm 5.0$ & 0.004 \\
\hline$V^{\prime} \mathrm{E} L \cdot \min ^{-1}$ & $57(47-78)$ & $44(40-54)$ & 0.04 \\
\hline Breathing reserve \%MVV & $11(6.6-26.3)$ & $22(1.0-40.7)$ & 0.60 \\
\hline VT $\mathrm{L}$ & $1.15(0.97-1.67)$ & $0.84(0.78-0.90)$ & 0.002 \\
\hline Respiratory rate breaths $\min ^{-1}$ & $49 \pm 8$ & $54 \pm 12$ & 0.21 \\
\hline HR beats $\cdot \min ^{-1}$ & $139 \pm 13$ & $128 \pm 23$ & 0.12 \\
\hline HR \% pred & $88 \pm 7$ & $78 \pm 12$ & 0.009 \\
\hline $\mathrm{V}^{\prime} \mathrm{O}_{2} / \mathrm{HR} \mathrm{mL} \cdot$ beat $^{-1} \cdot \mathrm{min}^{-1}$ & $10.8 \pm 3.1$ & $8.2 \pm 2.0$ & 0.02 \\
\hline$V^{\prime} \mathrm{O}_{2} / \mathrm{HR} \%$ pred & $107 \pm 11$ & $80 \pm 13$ & $<0.001$ \\
\hline $\mathrm{SpO}_{2} \%$ & $83 \pm 6$ & $79 \pm 8$ & 0.10 \\
\hline Borg dyspnoea score & $5(4-9)$ & $9(5-10)$ & 0.13 \\
\hline Borg leg discomfort score & $7(5-9)$ & $9(2-9)$ & 0.91 \\
\hline$\Delta \mathrm{IC}$ from rest $\mathrm{L}$ & $-0.01 \pm 0.27$ & $-0.02 \pm 0.12$ & 0.94 \\
\hline$\Delta \mathrm{IC}$ from rest $\%$ & $2.5 \pm 16$ & $1.4 \pm 9$ & 0.81 \\
\hline $\mathrm{V} / \mathrm{IC}$ & $0.80 \pm 0.13$ & $0.75 \pm 0.17$ & 0.40 \\
\hline \multicolumn{4}{|c|}{$\begin{array}{l}\text { Data are presented as mean } \pm \mathrm{SD} \text { or median (25th-75th percentile), unless otherwise stated. } V^{\prime} \mathrm{O}_{2} \text { : oxygen } \\
\text { production; } P \mathrm{ETCO}_{2} \text { : end-tidal carbon dioxide tension; RER: respiratory exchange rate; } V^{\prime} \mathrm{E} \text { : minute } \\
\text { ventilation; } V^{\prime} \mathrm{CO}_{2} \text { : carbon dioxide production; MVV: maximal voluntary ventilation; } V \mathrm{~T} \text { : tidal volume; HR: } \\
\text { heart rate; IC: inspiratory capacity; } \mathrm{SpO}_{2} \text { : arterial oxygen saturation measured by pulse oximetry. }\end{array}$} \\
\hline
\end{tabular}




\section{TABLE 4 Pulmonary function in patients with chronic hypersensitivity pneumonitis}

\begin{tabular}{|c|c|c|c|}
\hline & $V^{\prime} \mathrm{O}_{2} \geqslant 84 \%$ pred & $V^{\prime} \mathrm{O}_{2}<84 \%$ pred & p-value \\
\hline Subjects $\mathrm{n}$ & 14 & 14 & \\
\hline FVC L & $2.22 \pm 0.60$ & $1.85 \pm 0.66$ & 0.13 \\
\hline FVC $\%$ pred & $62(58-70)$ & $45(41-59)$ & 0.002 \\
\hline FEV 1 L & $1.88 \pm 0.45$ & $1.62 \pm 0.56$ & 0.20 \\
\hline FEV $1 \%$ pred & $70 \pm 13$ & $53 \pm 14$ & 0.003 \\
\hline FEV $1 / F V C$ & $0.86 \pm 0.06$ & $0.89 \pm 0.04$ & 0.14 \\
\hline FEV 1 FVC $\%$ pred & $106(105-113)$ & $108(105-115)$ & 0.54 \\
\hline FEF $25-75 \%$ & $101 \pm 29$ & $95 \pm 34$ & 0.65 \\
\hline TLC L & $3.67(3.00-4.17)$ & $2.92(2.43-3.59)$ & 0.14 \\
\hline TLC \% pred & $69(62-77)$ & $55(49-67)$ & 0.005 \\
\hline RV L & $1.33(1.10-1.52)$ & $1.15(0.98-1.38)$ & 0.25 \\
\hline RV \% pred & 78 (69-91) & $76(56-86)$ & 0.35 \\
\hline RV/TLC & $0.37 \pm 0.06$ & $0.41 \pm 0.07$ & 0.21 \\
\hline RV/TLC \% pred & $131 \pm 29$ & $151 \pm 45$ & 0.17 \\
\hline DLco \% pred & $50(38-71)$ & $33(26-45)$ & 0.01 \\
\hline \multicolumn{4}{|c|}{$\begin{array}{l}\text { Data are presented as mean } \pm \text { SD or median }\left(25 \text { th- } 75 \text { th percentile), unless otherwise stated. } V^{\prime} \mathrm{O}_{2} \text { : oxygen }\right. \\
\text { production; } \mathrm{FVC} \text { : forced vital capacity; } \mathrm{FEV} 1 \text { : forced expiratory volume in } 1 \mathrm{~s} \text {; } F E F 25-75 \% \text { : forced expiratory } \\
\text { flow at } 25-75 \% \text { of } F V C \text {; TLC: total lung capacity; RV: residual volume; DLCO: diffusing capacity of the lung } \\
\text { for carbon monoxide. }\end{array}$} \\
\hline
\end{tabular}

$\mathrm{p}=0.006$; sensitivity $92.9 \%$, specificity $64.3 \%$ ) and a DLCO (\% pred) of $30.0 \%$ (AUC $0.778,95 \%$ CI $0.60-0.96, p=0.02$; sensitivity $100 \%$, specificity $46.2 \%$ ) predicted normal exercise capacity (figure 3 ).

Table 5 compares responses to the SF-36 survey between cHP patients with normal and low exercise capacity. As expected, cHP patients with low exercise capacity had lower physical functioning scores ( $55 \pm 27$ versus $36 \pm 20, \mathrm{p}=0.05$ ) and a tendency towards lower scores in role limitation due to physical health $(\mathrm{p}=0.09)$ and vitality $(\mathrm{p}=0.06)$. No differences were observed in other domains.

\section{Discussion}

Our study aimed to evaluate the exercise capacity and the mechanisms underlying exercise limitation, including the development of $\mathrm{DH}$ in patients with cHP. Thus, we compared time course responses of the cardiopulmonary and metabolic systems with those of healthy controls. The main findings of our study were: 1) exercise capacity is lower in cHP patients than in healthy subjects; 2) ventilatory limitation and gas exchange impairment with increased dyspnoea were the major reasons for exercise cessation in $\mathrm{cHP}$ patients; 3) the prevalence of $\mathrm{DH}$ during exercise in cHP patients is low, and without clinically significant
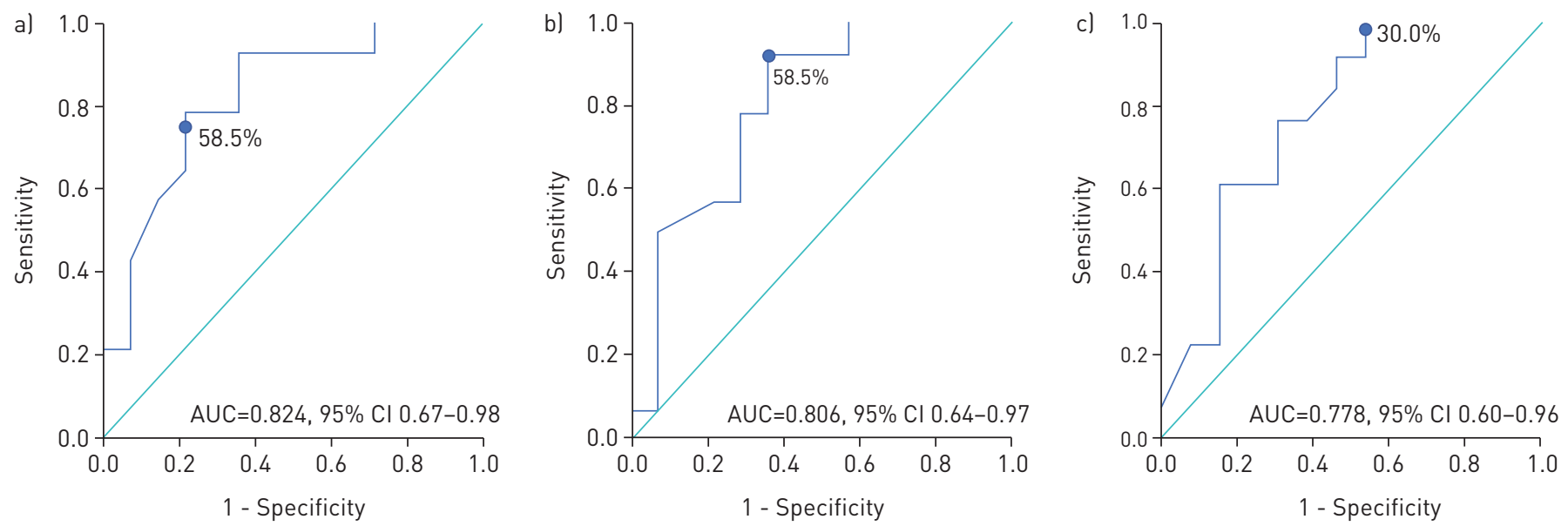

FIGURE 3 Receiver operating characteristic curve for a) forced vital capacity (\% predicted), b) total lung capacity (\% predicted) and c) diffusing capacity of the lung for carbon dioxide (\% predicted) for predicting normal exercise capacity in chronic hypersensitivity patients. AUC: area under the curve. 


\begin{tabular}{|c|c|c|c|}
\hline & $V^{\prime} \mathrm{O}_{2} \geqslant 84 \%$ pred & $V^{\prime} \mathrm{O}_{2}<84 \%$ pred & p-value \\
\hline Subjects $\mathrm{n}$ & 14 & 14 & \\
\hline \multicolumn{4}{|l|}{ Physical component scores } \\
\hline Physical functioning & $55 \pm 27$ & $36 \pm 20$ & $0.05^{*}$ \\
\hline Role limitation due to physical health & $50(0-75)$ & $0(0-25)$ & 0.09 \\
\hline Bodily pain & $79(45-100)$ & $68(48-83)$ & 0.43 \\
\hline General health & $63(39-90)$ & $45(34-69)$ & 0.15 \\
\hline \multicolumn{4}{|l|}{ Mental component scores } \\
\hline Role limitation due to emotional problems & $17(0-100)$ & $33(0-100)$ & 0.73 \\
\hline Vitality & $65 \pm 25$ & $50 \pm 14$ & 0.06 \\
\hline Emotional well-being & $70 \pm 21$ & $63 \pm 19$ & 0.38 \\
\hline Social functioning & $75 \pm 22$ & $69 \pm 25$ & 0.48 \\
\hline
\end{tabular}

repercussions; and 4) these findings during exercise are related to disease severity, and lung volumes and DLCO are good predictors of exercise limitation.

cHP patients had diminished exercise performance and lower $V^{\prime} \mathrm{O}_{2}$ compared to normal subjects, which was mainly due to ventilatory limitations and gas exchange impairment. Reduced exercise performance occurred in half of our patients. cHP patients presented with reduced breathing reserve and a greater $V^{\prime} \mathrm{E} / V^{\prime} \mathrm{CO}_{2}$ slope, oxygen desaturation in all but two patients and increases in $V^{\prime} \mathrm{E}$ based on respiratory rate, with a small increment of $V \mathrm{~T}$, which is in accordance with the restrictive pattern found in patients with ILD [31]. In heathy subjects, the expansion of $V T$ during exercise is accommodated within the most compliant, linear portion of the respiratory system's pressure-volume relationship [32] and the effort-displacement ratio remains relatively constant during exercise [33]. In cHP, as in other ILDs, the respiratory system's pressure-volume curve is contracted along its volume axis and is shifted downward and rightward, indicating increased elastic recoil pressure of the lung at any given lung volume [34, 35].

Severe dyspnoea, as demonstrated by higher Borg scores, was the dominant exercise-limiting symptom in these patients. cHP patients had a lower heart rate and an oxygen pulse than healthy subjects, probably caused by precocious interruption of exercise due to ventilatory limitation and/or oxygen desaturation, since no patient had evidence of cardiovascular limitation or pulmonary arterial hypertension, such as high $V^{\prime} \mathrm{E} / V^{\prime} \mathrm{CO}_{2}$ or low partial pressure of end-tidal carbon dioxide at the end of the exercise [36]. Although our protocol excluded patients with pulmonary hypertension, it is not possible to completely rule it out as a possible limiting factor as hypersensitivity pneumonitis may present pulmonary hypertension even in the presence of a normal echocardiograph [10,37, 38]. Additionally, CPET is not a reliable predictor of pulmonary hypertension in these patients [10].

Two patients in our study had significant chronotropic and ventilatory reserve without other reasons for exercise limitation, justifying peripheral muscle dysfunction. However, we emphasise that besides the high prevalence of corticosteroids in our cohort and its potential role in exercise limitation as previously described in ILD patients [39], 93\% of our patients interrupted exercise due to dyspnoea, with diverse indexes of ventilatory limitation, which reinforces that peripheral muscle dysfunction was not the prevalent mechanism for exercise cessation in these patients.

Although the prevalence of $\mathrm{DH}$ in our sample was $18 \%$, no difference in IC variation was observed between healthy and cHP patients or between cHP subgroups (normal versus low exercise capacity), suggesting that $\mathrm{DH}$ is not a relevant mechanism for the exercise limitations in these patients. The explanation for IC variation in cHP remains to be elucidated.

One potential mechanism may be due to $\mathrm{DH}$ per se secondary to small airway involvement. Although PFTs showed restrictive lung disease in all but two patients, the RV/TLC ratio in our entire cohort was $141 \pm 38 \%$ pred, which could suggest concomitant small airway involvement. Also, besides signs of small airway involvement in all biopsied patients and the presence of mosaic attenuation on CT in 54\% of the entire cohort, patients had severe restrictive impairment, and only three patients had physiological signs of air trapping. The exact prevalence of obstructive disorder in cHP is unknown; it is higher in hot tub lung disease [40] and farmer's lung [41], but also reduces progressively the longer the diagnosis, probably 
reflecting the evolution of fibrosis [42]. Our patients had a median time after diagnosis of 24 months, which could have contributed to our results. It is likely that in our cohort of patients the influence of $\mathrm{DH}$ is milder, and the increased elastic recoil component prevails functionally and during exercise; besides, traction bronchiectasis may have attenuated air trapping caused by airway lesions. In patients with milder restriction and severe air trapping, DH may have a greater influence in exercise limitation. Due to the small number of patients with $\mathrm{DH}$, we could not explore lung function parameters or CPET data to determine predictive data for its occurrence.

A second mechanism may be mechanical factors and decreased inspiratory muscle performance. We observed a tendency towards higher $V \mathrm{~T} / \mathrm{IC}$ in $\mathrm{cHP}$ patients $(\mathrm{p}=0.06)$. In this context, a limitation of increased IC may have increased elastic recoil in restrictive lung disease. In these patients, increased breathing effort and respiratory muscle fatigue at greater $V^{\prime} \mathrm{E}$ may be an explanation for IC reduction. Due to the exploratory nature of our study, and lack of oesophageal pressure measurements, the role of this mechanism remains to be fully elucidated.

ILDs can present significant airway involvement and DH can be one of the mechanisms for exercise limitation, such as in patients with LAM [15]. In pulmonary Langerhans cell hystiocytosis patients, the "air trapping" component seemed to limit patients during exercise, as assessed by a lower FEV1/FVC ratio in patients with impaired exercise capacity; however, flow-volume loops were not performed in this study [43]. In sarcoidosis, in which ventilatory and cardiocirculatory impairment may occur, inspiratory manoeuvers during CPET have not been assessed. In LAM, DH had an intrinsic relationship with airway obstruction detected by pulmonary function tests. In ILD, we postulate that DH occurrence might also have a close relationship with airway obstruction, which may explain the low prevalence of DH in our study.

cHP patients with low exercise capacity were unable to increase their tidal ventilation compared to patients with normal exercise capacity, without differences in oxygenation, respiratory rate or breathing reserve. Also, breathing reserve alone was not a good predictor of ventilatory limitation in these patients, as previously shown in mild COPD [44]. Thus, the main factor for low exercise capacity in these patients seems to be disease severity assessed by PFTs parameters. Patients with normal exercise capacity had increased FVC \%, FEV1 \%, TLC \% and DLCO \%. For the first time, we could accurately determine the values of PFTs and predict worse exercise capacity in cHP. Based on the ROC curve analysis, the best predictor for worse exercise capacity was FVC (\% pred), and the value with the highest accuracy was $58.5 \%$, followed by a TLC of $58.5 \%$ pred and a DLCO of $30 \%$ pred. FVC is an easily available parameter in clinical practice. In the context of ILDs, functional tests, especially during exertion, can be important tools for treatment evaluation, follow-up and prognosis. However, the current literature is extrapolated from patients with idiopathic pulmonary fibrosis (IPF), with submaximal tests such as the 6-min walk distance $[45,46]$. To our knowledge, our study is the first to characterise functional data with preserved exercise capacity in cHP patients.

According to previous publications, a low DLCO, measured at rest, is the most significant predictor of exertional hypoxaemia in previous publications with fibrotic ILD; of note, fibrotic ILD patients have more severe exertional hypoxaemia than patients with COPD for a given degree of impairment in DLCO [47]. In ILD patients, it is also known that a substantial number of patients with normal DLCO have increased desaturation at maximal exercise, suggesting that normal DLCO at rest may be an inappropriate predictor of abnormal pulmonary gas exchange during exercise. We strongly believe that in exercise, unequal ventilation/perfusion rates in fibrotic lung parenchyma and low ventilatory efficiency (high $V^{\prime} \mathrm{E} / V^{\prime} \mathrm{CO}_{2}$ ) can contribute to a significant oxygen desaturation besides the degree of DLCO impairment measured at rest. Of note, it is also important to emphasise that although we hypothetically excluded pulmonary hypertension in our patients according to the study protocol, it is still possible it could have contributed to additional desaturation in our study.

cHP patients with low exercise capacity had lower physical functioning scores and a tendency towards lower scores in physical health and vitality. Other domains did not have significant differences between cHP groups. Studies assessing quality of life (QoL) in cHP are still emerging. A previous study reported that cHP patients had worse QoL scores when compared with IPF patients and the severity of dyspnoea and fatigue were the strongest factors associated with a poor QoL [48]. Our study confirmed that worse exercise capacity significantly impacted on physical score domains. However, other factors, such as socioeconomic support, physical and mental comorbidities, and cultural differences could also have a greater impact in other domains. Since QoL is increasingly being considered as a patient-centred outcome measure in ILD clinical trials [48-50], interventions such as rehabilitation programmes might significantly impact QoL in these patients.

Our study has some limitations that need to be addressed. Due to our strict inclusion criteria, our sample size was relatively small. Furthermore, our patient cohort was composed of patients with a low smoking 
burden. Heavy smokers were intentionally excluded to avoid confounding our results due to small airway involvement associated with smoking. Also, our cohort is not comprised of patients with obstructive disease and our results can only be extrapolated to patients with restrictive disease, which can explain the low prevalence of DH. Finally, our cohort did not include patients with farmer's lung, in which the presence of emphysema is classically described [51]. Studies with patients with subacute hypersensitivity pneumonitis, cHP with obstructive disease and farmer's lung are needed to elucidate the mechanisms involved in reduced exercise capacity in such different populations.

In conclusion, reduced exercise capacity was prevalent in cHP patients, occurring in $50 \%$ of our cohort. The primary causes of exercise limitations were found in ILD, such as ventilatory limitations and gas exchange impairment with augmented dyspnoea. Despite significant involvement of small airways in cHP patients, DH is infrequent during CPET, and without clinical repercussions. The main factor for preserved exercise capacity in cHP is the ability to increase tidal ventilation, which is associated with preserved lung function parameters. Patients with exercise impairment had lower physical functioning scores. Further studies are necessary in cHP patients to fully understand the mechanisms involved in the development of $\mathrm{DH}$, and the role of interventions such as pulmonary rehabilitation in exercise capacity and QoL improvement.

Acknowledgements: The authors thank colleagues from the Pulmonary Function and Exercise Physiology Laboratory and from the Interstitial Lung Disease outpatient clinic (Pulmonary Division, Heart Institute (InCor), University of Sâo Paulo Medical School, Sâo Paulo, Brazil) for their collaboration in this study.

Author contributions: O.M. Dias was responsible for the study conception, data collection, data analysis and interpretation, and drafting the article. J.G. Ferreira and L.Z. Cardenas were responsible for data collection. B.G. Baldi, C.R.R. Carvalho, F. Pennati, C. Saltio and A. Aliverti were responsible for critical revision of the article. A.L. Pereira de Albuquerque was responsible for the study design and final approval of the version to be published. All authors have read and approved the final manuscript.

Conflict of interest: Carlos Roberto Ribeiro Carvalho reports personal fees from Boehringer Ingelheim, outside the submitted work.

Support statement: Funding was received from the European Respiratory Society (Fellowship STRTF 2015). Funding information for this article has been deposited with the Crossref Funder Registry.

\section{References}

1 Lacasse Y, Girard M, Cormier Y. Recent advances in hypersensitivity pneumonitis. Chest 2012; 142: 208-217.

2 Lacasse Y, Selman M, Costabel U, et al. Clinical diagnosis of hypersensitivity pneumonitis. Am J Respir Crit Care Med 2003; 168: 952-958.

3 Lacasse Y, Selman M, Costabel U, et al. Classification of hypersensitivity pneumonitis: a hypothesis. Int Arch Allergy Immunol 2009; 149: 161-166.

4 Vasakova M, Morell F, Walsh S, et al. Hypersensitivity pneumonitis: perspectives in diagnosis and management. Am J Respir Crit Care Med 2017; 199: 680-689.

5 Wang $\mathrm{P}$, Jones KD, Urisman A, et al. Pathologic findings and prognosis in a large prospective cohort of chronic hypersensitivity pneumonitis. Chest 2017; 152: 502-509.

6 Guerrero Zuniga S, Sanchez Hernandez J, Mateos Toledo H, et al. Small airway dysfunction in chronic hypersensitivity pneumonitis. Respirology 2017; 22: 1637-1642.

7 Silva CI, Churg A, Muller NL. Hypersensitivity pneumonitis: spectrum of high-resolution CT and pathologic findings. AJR Am J Roentgenol 2007; 188: 334-344.

8 Dias OM, Baldi BG, Pennati F, et al. Computed tomography in hypersensitivity pneumonitis: main findings, differential diagnosis and pitfalls. Expert Rev Respir Med 2018; 12: 5-13.

9 Silva CI, Muller NL, Lynch DA, et al. Chronic hypersensitivity pneumonitis: differentiation from idiopathic pulmonary fibrosis and nonspecific interstitial pneumonia by using thin-section CT. Radiology 2008; 246: 288-297.

10 Oliveira RK, Pereira CA, Ramos RP, et al. A haemodynamic study of pulmonary hypertension in chronic hypersensitivity pneumonitis. Eur Respir J 2014; 44: 415-424.

11 Schwaiblmair M, Beinert T, Vogelmeier C, et al. Cardiopulmonary exercise testing following hay exposure challenge in farmer's lung. Eur Respir J 1997; 10: 2360-2365.

12 O'Donnell DE, Revill SM, Webb KA. Dynamic hyperinflation and exercise intolerance in chronic obstructive pulmonary disease. Am J Respir Crit Care Med 2001; 164: 770-777.

13 O'Donnell DE, Travers J, Webb KA, et al. Reliability of ventilatory parameters during cycle ergometry in multicentre trials in COPD. Eur Respir J 2009; 34: 866-874.

14 Calverley PM, Koulouris NG. Flow limitation and dynamic hyperinflation: key concepts in modern respiratory physiology. Eur Respir J 2005; 25: 186-199.

15 Baldi BG, Albuquerque AL, Pimenta SP, et al. Exercise performance and dynamic hyperinflation in lymphangioleiomyomatosis. Am J Respir Crit Care Med 2012; 186: 341-348.

16 Marciniuk DD, Watts RE, Gallagher CG. Dead space loading and exercise limitation in patients with interstitial lung disease. Chest 1994; 105: 183-189.

17 O'Donnell DE, Chau LK, Webb KA. Qualitative aspects of exertional dyspnea in patients with interstitial lung disease. J Appl Physiol (1985) 1998; 84: 2000-2009.

18 Marciniuk DD, Sridhar G, Clemens RE, et al. Lung volumes and expiratory flow limitation during exercise in interstitial lung disease. J Appl Physiol (1985) 1994; 77: 963-973. 
19 Ware JE Jr. SF-36 health survey update. Spine (Phila Pa 1976) 2000; 25: 3130-3139.

20 Ciconelli RM, Ferraz M, Santos W, et al. Tradução para língua portuguesa e validação do questionário genérico de avaliação de qualidade de vida SF-36 (Brasil SF-36). Rev Bras Reumatol 1999; 39: 143-150.

21 Macintyre N, Crapo RO, Viegi G, et al. Standardisation of the single-breath determination of carbon monoxide uptake in the lung. Eur Respir J 2005; 26: 720-735.

22 Miller MR, Hankinson J, Brusasco V, et al. Standardisation of spirometry. Eur Respir J 2005; 26: 319-338.

23 Wanger J, Clausen JL, Coates A, et al. Standardisation of the measurement of lung volumes. Eur Respir J 2005; 26: 511-522.

24 Neder JA, Andreoni S, Peres C, et al. Reference values for lung function tests. III. Carbon monoxide diffusing capacity (transfer factor). Braz J Med Biol Res 1999; 32: 729-737.

25 Pereira CA, Sato T, Rodrigues SC. New reference values for forced spirometry in white adults in Brazil. $J$ Bras Pneumol 2007; 33: 397-406.

26 Neder JA, Nery LE, Castelo A, et al. Prediction of metabolic and cardiopulmonary responses to maximum cycle ergometry: a randomised study. Eur Respir J 1999; 14: 1304-1313.

27 American Thoracic Society, American College of Chest Physicians. ATS/ACCP Statement on cardiopulmonary exercise testing. Am J Respir Crit Care Med 2003; 167: 211-277.

28 Borg GA. Psychophysical bases of perceived exertion. Med Sci Sports Exerc 1982; 14: 377-381.

29 Vogiatzis I, Georgiadou O, Golemati S, et al. Patterns of dynamic hyperinflation during exercise and recovery in patients with severe chronic obstructive pulmonary disease. Thorax 2005; 60: 723-729.

30 Stubbing DG, Pengelly LD, Morse JL, et al. Pulmonary mechanics during exercise in subjects with chronic airflow obstruction. J Appl Physiol Respir Environ Exerc Physiol 1980; 49: 511-515.

31 Guenette JA, Chin RC, Cory JM, et al. Inspiratory capacity during exercise: measurement, analysis, and interpretation. Pulm Med 2013; 2013: 956081.

32 Jensen D, Ofir D, O'Donnell DE. Effects of pregnancy, obesity and aging on the intensity of perceived breathlessness during exercise in healthy humans. Respir Physiol Neurobiol 2009; 167: 87-100.

33 O’Donnell DE, Hong HH, Webb KA. Respiratory sensation during chest wall restriction and dead space loading in exercising men. J Appl Physiol (1985) 2000; 88: 1859-1869.

34 Yernault JC, de Jonghe M, de Coster A, et al. Pulmonary mechanics in diffuse fibrosing alveolitis. Bull Physiopathol Respir (Nancy) 1975; 11: 231-244.

35 Gibson GJ, Pride NB. Pulmonary mechanics in fibrosing alveolitis: the effects of lung shrinkage. Am Rev Respir Dis 1977; 116: 637-647.

36 Armstrong HF, Schulze PC, Bacchetta M, et al. Impact of pulmonary hypertension on exercise performance in patients with interstitial lung disease undergoing evaluation for lung transplantation. Respirology 2014; 19: 675-682.

37 Arcasoy SM, Christie JD, Ferrari VA, et al. Echocardiographic assessment of pulmonary hypertension in patients with advanced lung disease. Am J Respir Crit Care Med 2003; 167: 735-740.

38 Nathan SD, Shlobin OA, Barnett SD, et al. Right ventricular systolic pressure by echocardiography as a predictor of pulmonary hypertension in idiopathic pulmonary fibrosis. Respir Med 2008; 102: 1305-1310.

39 Panagiotou M, Polychronopoulos V, Strange C. Respiratory and lower limb muscle function in interstitial lung disease. Chron Respir Dis 2016; 13: 162-172.

40 Hanak V, Golbin JM, Ryu JH. Causes and presenting features in 85 consecutive patients with hypersensitivity pneumonitis. Mayo Clin Proc 2007; 82: 812-816.

41 Lalancette M, Carrier G, Laviolette M, et al. Farmer's lung. Long-term outcome and lack of predictive value of bronchoalveolar lavage fibrosing factors. Am Rev Respir Dis 1993; 148: 216-221.

42 Selman-Lama M, Perez-Padilla R. Airflow obstruction and airway lesions in hypersensitivity pneumonitis. Clin Chest Med 1993; 14: 699-714.

43 Rolland-Debord C, Fry S, Giovannelli J, et al. Physiologic determinants of exercise capacity in pulmonary Langerhans cell histiocytosis: a multidimensional analysis. PLoS One 2017; 12: e0170035.

44 Elbehairy AF, Ciavaglia CE, Webb KA, et al. Pulmonary gas exchange abnormalities in mild chronic obstructive pulmonary disease. Implications for dyspnea and exercise intolerance. Am J Respir Crit Care Med 2015; 191: $1384-1394$.

45 Lederer DJ, Arcasoy SM, Wilt JS, et al. Six-minute-walk distance predicts waiting list survival in idiopathic pulmonary fibrosis. Am J Respir Crit Care Med 2006; 174: 659-664.

46 du Bois RM, Albera C, Bradford WZ, et al. 6-Minute walk distance is an independent predictor of mortality in patients with idiopathic pulmonary fibrosis. Eur Respir J 2014; 43: 1421-1429.

47 Du Plessis JP, Fernandes S, Jamal R, et al. Exertional hypoxemia is more severe in fibrotic interstitial lung disease than in COPD. Respirology 2018; 23: 392-398.

48 Olson AL, Brown KK, Swigris JJ. Understanding and optimizing health-related quality of life and physical functional capacity in idiopathic pulmonary fibrosis. Patient Relat Outcome Meas 2016; 7: 29-35.

49 Lammi MR, Baughman RP, Birring SS, et al. Outcome measures for clinical trials in interstitial lung diseases. Curr Respir Med Rev 2015; 11: 163-174.

50 Russell AM, Sprangers MA, Wibberley S, et al. The need for patient-centred clinical research in idiopathic pulmonary fibrosis. BMC Med 2015; 13: 240.

51 Erkinjuntti-Pekkanen R, Rytkonen H, Kokkarinen JI, et al. Long-term risk of emphysema in patients with farmer's lung and matched control farmers. Am J Respir Crit Care Med 1998; 158: 662-665. 